\title{
ESTRANHOS NA HISTÓRIA DA LITERATURA BRASILEIRA: ELIZABETH BISHOP COMO MEDIADORA CULTURAL
}

\author{
EXTRAÑOS EN LA HISTORIA DE LA LITERATURA BRASILEÑA: \\ ELIZABETH BISHOP COMO MEDIADORA CULTURAL
}

\section{STRANGERS IN THE HISTORY OF BRAZILIAN LITERATURE: ELIZABETH BISHOP AS A CULTURAL MEDIATOR}

Elisabete da Silva Barbosa Universidade do Estado da Bahia, Caetité, Bahia, Brasil

ID Lattes: http://lattes.cnpq.br/6350392247932607

ORCID: https://orcid.org/0000-0002-6419-822

Endereço eletrônico: elisabete_barbosa@hotmail.com

DOI: $10.22481 /$ rbba.v10i01.8778

\section{RESUMO}

A história literária que, no passado, se pensava totalizadora, tem sido entendida na contemporaneidade como atividade provisória e lacunar, a exemplo de Brazil 2001: A revisionary History of Brazilian Literature and Culture. Trata-se de coletânea composta por ensaios em língua inglesa escritos por autores, em sua maioria, brasileiros. Os textos revisam diferentes concepções da identidade nacional, tomando esse termo na acepção de totalidade incompleta e lacunar (CHAUÍ, 2000). Dado o panorama mais amplo, buscamos refletir sobre os critérios para a inserção de estrangeiros que, na condição de mediadores culturais, contribuem para a escrita da história da literatura brasileira. Nessa publicação, Paulo Henriques Britto (2000) redige o texto Elizabeth Bishop as cultural intermediary, com o qual estabelecemos diálogo a fim de compreender o papel de intermediária cultural desempenhado por Bishop. 
Palavras-chave: Elizabeth Bishop; História da Literatura; Intermediação Cultural.

\title{
RESUMEN
}

La historia literaria que, en el pasado, se pensaba totalizadora, se ha entendido en la época contemporánea como una actividad provisional y lacunar, como Brasil 2001: una historia revisionista de la literatura y la cultura brasileñas. Se trata de una colección de ensayos en inglés escritos por autores, en su mayoría brasileños. Los textos revisan diferentes concepciones de la identidad nacional, utilizando este término en el sentido de totalidad incompleta y lacunar (CHAUí, 2000). Ante el panorama más amplio, buscamos reflexionar sobre los criterios para la inserción de extranjeros que, como mediadores culturales, contribuyen a la redacción de la historia de la literatura brasileña. En esta publicación, Paulo Henriques Britto (2000) escribe el texto Elizabeth Bishop as cultural intermediary, con el que establecemos un diálogo para comprender el papel del intermediaria cultural interpretado por Bishop.

Palabras clave: Elizabeth Bishop; Historia de la Literatura; Intermediación Cultural.

\begin{abstract}
The literary history that, in the past, was thought to be totalizing, has been understood in contemporary times as a provisional and lacunar activity, as in the case of Brazil 2001: A revisionary History of Brazilian Literature and Culture. It is a collection of essays in English written mostly by Brazilian authors. The texts review different conceptions of national identity, using this term in the sense of incomplete and lacunae totality (CHAUÍ, 2000). Given the broader picture, we seek to reflect on the criteria for the insertion of foreigners who, as cultural mediators, contribute to the writing of the history of Brazilian literature. In this publication, Paulo Henriques Britto (2000) writes the text Elizabeth Bishop as cultural intermediary, in which we establish dialogue in order to understand the role of cultural intermediary played by Bishop.
\end{abstract}

Keywords: Elizabeth Bishop; History of Literature; Cultural intermediation. 


\section{ESTRANHOS NA HSTÓRIA DA LITERATURA BRASILEIRA: ELIZABETH BISHOP COMO MEDIADORA CULTURAL}

\section{INTRODUÇÃO}

O propósito deste artigo é, em um primeiro momento, discutir a respeito do fazer historiográfico e perpassar algumas concepções de história literária desenvolvidas no Brasil. Em um segundo momento, estabelecer contraposição entre a concepção tradicional de história literária e a compreensão do fazer historiográfico mais recente, abordado na contemporaneidade como atividade provisória e lacunar.

Nesse contexto, discorreremos a respeito da publicação do Center for Portuguese Studies and Culture da Universidade de Massachusetts intitulada Brazil 2001: A revisionary History of Brazilian Literature and Culture. Trata-se de coletânea organizada por João Cezar de Castro Rocha (2000), composta por ensaios em língua inglesa escritos por diversos críticos, em sua maioria, brasileiros.

Após tal contextualização, destacamos um caso particular, o da inclusão da autora norteamericana Elizabeth Bishop (1911-1979) em Brazil 2001. O objetivo é compreender a percepção do crítico Paulo Henriques Britto (2000) - tradutor da obra de Elizabeth Bishop no Brasil - a respeito da atividade mediadora exercida pela escritora e, a partir disso, realizar uma leitura do texto de Britto (2000) ao lado de outros estudos realizados sobre a obra de Bishop, a partir do acionamento dos conceitos de paradigma indiciário e de circularidade cultural de Carlos Ginzburg (2007). Essa trajetória de análise textual nos habilita a refletir sobre o fato de a identidade cultural brasileira poder ser pensada a partir dos escritos dessa autora - e, por conseguinte, de outros estrangeiros -, como assevera Rocha (2000) em seu texto introdutório de Brazil 2001.

\section{DESENVOLVIMENTO DA HISTÓRIA DA LITERATURA BRASILEIRA}

Embora o primeiro livro de história literária, de autoria de Silvio Romero (1888), tenha surgido somente em fins do século XIX, já havia uma preocupação de agrupar textos produzidos no Brasil ou por brasileiros situados em Portugal, a fim de classificá-los sob o signo de uma nacionalidade brasileira. Apresentados em forma de resumos, bosquejos ou florilégios, tinham como objetivo a compilação da literatura em língua portuguesa.

Os primeiros estrangeiros que se ocuparam com esse assunto classificaram a literatura brasileira como apêndice daquela produzida em Portugal, pois tomavam a língua como fator de 


\section{ESTRANHOS NA HSTÓRIA DA LITERATURA BRASILEIRA: ELIZABETH BISHOP COMO MEDIADORA CULTURAL}

integração dos textos literários. Esse quadro começou a mudar com Ferdinand Denis (2018 [1826]), que propôs a substituição do fator linguístico pelo geográfico (1826), caminho para o desenvolvimento do olhar crítico sobre a literatura e para a sistematização dos textos brasileiros.

O Resumo da História literária de Portugal seguido do Resumo da História literária do Brasil tornou-se texto significativo não somente para críticos brasileiros, mas também para escritores de textos literários, uma vez que Denis (2018 [1826]) afirmou ser este um país livre tanto no aspecto político quanto no literário. É válido lembrar a condição do Brasil de recémindependência e a necessidade de criação de signos que atestassem a nova condição. Nessa oportunidade, Denis (2018 [1826]) apontou o direcionamento que essa literatura deveria tomar, indicando um modelo que pôs a cor local na agenda dos escritores brasileiros.

Somente em fins do século XIX e início do XX as histórias da literatura nacional começaram a ser escritas de forma independente de Portugal. Moldadas pelo positivismo e evolucionismo, ocupavam-se com o estabelecimento de critérios e discussões a respeito de conceitos, já que estavam ancoradas em teorias específicas. Nesse momento, elegeram o método histórico para a investigação dos objetos literários e para a formação do cânone. $\mathrm{O}$ primeiro desses textos data do final do século XIX, quando Silvio Romero (1888) publicou a História da Literatura Brasileira. Quase duas décadas depois, José Veríssimo (1916) lançou uma obra com título idêntico. Ainda nesse período, destacamos o projeto não concluído de Tristão de Alencar Araripe Junior (1848-1911), com a produção de textos esparsos que adotavam a vertente da história da literatura alemã. $\mathrm{O}$ fator integrador dos textos privilegiados em seus escritos foi o meio, elemento importante para a criação do conceito de obnubilação brasílica. Com isso, Araripe Junior pôde considerar as obras literárias dos primeiros cronistas como literatura brasileira. Em suas palavras, esses escritores teriam passado por uma transformação ao primeiro contato com o meio. A obnubilação brasílica, lei norteadora de seus trabalhos, seria validada por seu projeto, voltado a desenvolver perfis literários.

A partir da segunda metade do século XX, tais trabalhos foram relidos à luz de novos instrumentais teóricos. À essa altura, os intelectuais brasileiros já haviam aprendido a olhar o próprio passado e a investigar as produções nacionais e, com elas, dialogar, tendo em vista o objetivo de elaborar uma tradição historiográfica - o que ainda estava na agenda do dia - e de repensar a construção de um cânone que, a partir dos escritos de Veríssimo, já se sabia flexível e em constante construção. 


\section{ESTRANHOS NA HSTÓRIA DA LITERATURA BRASILEIRA: ELIZABETH BISHOP COMO MEDIADORA CULTURAL}

Em 1959, Antonio Candido publicou a Formação da literatura brasileira: momentos decisivos, com ênfase no processo formativo da literatura. Com essa nova forma de abordagem, Candido (2012 [1959]) operou um corte no modo tradicional do fazer historiográfico, o qual costumava buscar uma origem fixa ou mítica para a nacionalidade e, por conseguinte, para o surgimento da literatura.

Em 1968, Afrânio Coutinho organizou A literatura no Brasil. Distribuída em seis volumes, contou com a colaboração de intelectuais especialistas. As temáticas, assim como a organização do livro, seguiram o viés estético, o que se pode observar na divisão por estilos, com foco na obra e não em fatores externos. Trata-se de um fazer que privilegiou a discussão da metodologia adotada e dos princípios diretores da historiografia. Para o estabelecimento do ponto inicial da literatura brasileira, Coutinho (2004 [1968]) revisitou os textos de Araripe Júnior e, a partir do conceito de obnubilação brasílica, também elegeu a literatura produzida no período colonial como ponto de partida, considerando-a nacional.

As histórias da literatura acima comentadas são tomadas como amostragens de que a teoria norteadora do fazer historiográfico interfere no modo como um texto histórico é elaborado e organizado e, por conseguinte, repercute na eleição dos autores canônicos. A partir do século XX, o fazer historiográfico passou a ser impactado pelos pressupostos da Nova História, corrente que considera como fonte não mais apenas os documentos oficiais. Soma-se a isso o fato de a própria linguagem passar a ser percebida em sua relatividade.

Segundo Ricoeur (2007), devemos desconfiar da suposta transparência da linguagem em relação a um referente, pensamento que muda radicalmente a forma de se pensar a história e, por conseguinte, a história literária. Altera-se, portanto, a relação entre a produção histórica e a verdade. Com isso, o cânone que foi se estabelecendo ao longo do tempo tornou-se questionável, especialmente com o surgimento dos Estudos Culturais, campo de investigação que denuncia seu caráter de exclusão.

Influenciado por tais discussões, observamos que o texto de Antonio Cândido (2012 [1959]) - tanto quanto o de Harold Bloom (2010 [1994]) - orienta-se pela ideia de que a eleição do cânone é pessoal e, portanto, depende do ponto de vista e dos critérios adotados por quem o organiza. Quanto à proposta de Afrânio Coutinho (2004 [1968]), sublinhamos a divisão da tarefa historiográfica entre vários intelectuais especialistas como um avanço.

Adotando orientação similar, a publicação do Center for Portuguese Studies and Culture da Universidade de Massachusetts Brazil 2001: A revisionary History of Brazilian Literature 


\section{ESTRANHOS NA HSTÓRIA DA LITERATURA BRASILEIRA: ELIZABETH BISHOP COMO MEDIADORA CULTURAL}

and Culture foi organizada por João Cezar de Castro Rocha (2000) com o patrocínio da Universidade do Estado do Rio de Janeiro. Embora estruturada por ensaios em língua inglesa, a autoria é composta, em sua maior parte, por pesquisadores brasileiros que contribuíram com estudos críticos a respeito de ícones da literatura e da cultura considerados, nessa nova proposta de história da literatura, relevantes de terem seus pensamentos e representações a respeito do Brasil ali reunidos.

No texto introdutório de título There is no Brazil, Rocha (2000) apresenta o poema Hino Nacional de Carlos Drummond de Andrade (2013) para demonstrar, por meio da produção poética, que o Brasil não pode ser apreendido e representado em sua totalidade. Nos versos finais - “[...] Nosso Brasil é no outro mundo. Este não é o Brasil. / Nenhum Brasil existe. E acaso existirão os brasileiros?" - Drummond convida a uma interpretação em que o Brasil perde sua unidade enquanto signo representativo, pois "[...] não pode ser reduzido à imagem oficial do país" (ROCHA, 2000, p. 18, tradução nossa).

O Brasil seria, desse modo, de um signo que, como qualquer outro, escapa a uma suposta representação totalizadora, o que levou Rocha (2000) a mencionar a existência de um paradoxo: “O 'Brasil' oficial não coincide com o Brasil. Talvez porque não exista um Brasil e, ao mesmo tempo, seja o próprio Brasil que revele a sua não existência”. Tal ideia se articula a uma das palavras presentes no título, revisionary.

A respeito desse vocábulo, Rocha (2000) revisita a reflexão proposta Tamen, quem afirma que, etimologicamente, as palavras theoria e revisio correlacionam-se às ideias de phantasia e phantasma pois, para os gregos, um pronunciamento teórico implicava re-vision, isto é, um discurso proferido por um theoros (função pública) a fim de reportar um evento e, desse modo, atestar sua real ocorrência. Tal ato implicava a existência de ouvintes que não presenciaram o evento abordado pelo theoroi. O teórico, nesse sentido, estava investido de credibilidade e seu pronunciamento funcionava como ato suplementar que conferia veracidade à re-visão do evento (ROCHA, 2000).

$\mathrm{O}$ ato de re-visão pode ser então percebido por meio de duas abordagens distintas: se, por um lado, o pronunciamento do theoros é fantasmagórico, sendo o fantasma uma "imagem secundária", por outro, do ponto de vista do ouvinte da teoria, os "[...] fantasmas re-visados são 'secundários à imagem nenhuma', pois surgem simultaneamente ao discurso teórico" (ROCHA, 2000, p. 19, tradução nossa). Assim, a proposta de Brazil 2001 alinha-se com a ideia contida na palavra revisionary, pois seus leitores não podem ter acesso ao evento original, mas 


\section{ESTRANHOS NA HSTÓRIA DA LITERATURA BRASILEIRA: ELIZABETH BISHOP COMO MEDIADORA CULTURAL}

acessá-lo somente por meio de uma representação cujo referente, a priori, inexiste, o que configura tal evento como algo não testemunhado.

Rocha (2000, p. 19, tradução nossa) ainda lembra que, “[...] no que diz respeito à história cultural e literária, não há evento original ao qual as narrativas possam se referir” e que a performance teórica deve operar uma inversão, fornecendo uma visão retrospectiva. Nesse sentido, o pesquisador afirma que o paradoxo proposto por Drummond no poema Hino Nacional não pode ser resolvido.

Tal contradição pode ser elucidada com o pensamento de Homi Bhabha (2007), para quem a nação é narração e opera exclusões por não ser capaz de dar conta da complexidade de que é formada. Ainda pontua que a ideia de nação requer de seus membros o esquecimento de alguns eventos pois, se fossem permanentemente lembrados, inviabilizariam a ideia de coletividade que subjaz o sentimento de pertença.

O esquecimento envolvido na formação de uma nação torna-se mais um elemento definidor do caráter lacunar da história. Assim, podemos afirmar que a questão proposta por Drummond (2013) em Hino Nacional remete não a um problema especificamente brasileiro, mas a "[...] uma questão teórica relacionada à constituição da sociedade moderna" (ROCHA, 2000, p. 21, tradução nossa).

É nesses termos que a palavra revisionary serve como elo unificador dos textos de Brazil 2001 e seu objetivo é funcionar como uma história da literatura e da cultura que se sabe provisória. Seus textos revisitam diferentes concepções de identidade nacional, tomando esse conceito na acepção explicada por Marilena Chauí (2000) como totalidade incompleta e lacunar. De natureza ensaística, os textos não se propõem a encontrar conclusões a respeito de uma identidade brasileira, mas a apresentar as nuances percebidas e registradas por diversos autores, sejam literários ou não, que desenvolveram objetos estéticos ou um pensamento mais acurado a respeito da cultura brasileira.

\section{INTERMEDIÁRIOS CULTURAIS}

Dada a construção teórica subjacente ao fazer historiográfico de Brazil 2001, torna-se possível compreender o lugar das representações do Brasil propostas por estrangeiros para se pensar a literatura e a cultura na contemporaneidade. Nessa publicação, destacam-se os nomes de Pero Vaz de Caminha, José de Anchieta, Padre Antônio Vieira, Ferdinand Denis, Lévi- 


\section{ESTRANHOS NA HSTÓRIA DA LITERATURA BRASILEIRA: ELIZABETH BISHOP COMO MEDIADORA CULTURAL}

Strauss, Jean Baptiste Debret, Otto Maria Carpeaux, Roger Bastide, Daniel Defoe, Stefan Zweig, Ziembinski, Vilém Flusser e Elizabeth Bishop.

O estrangeiro tem, como atesta essa publicação, contribuído, de alguma forma, para uma escrita da história da literatura e da cultura brasileira. De fato, o olhar estrangeiro foi o primeiro a fornecer uma caracterização do Brasil e do brasileiro e, portanto, podemos considerar o encontro com a diferença elemento mobilizador da representação de identidades.

Com o intuito de discutir a condição do estrangeiro e, mais especificamente, de Elizabeth Bishop como mediadora cultural, tecemos algumas considerações sobre o texto de Paulo Henriques Britto (2000) intitulado Elizabeth Bishop as Cultural Intermediary. A partir disso, focalizarmos o arquivo da autora para entendê-lo como locus de uma memória eivada do aspecto fantasmagórico assinalado por Rocha (2000), pois passaram pelo filtro da linguagem que, em seu papel de signo, está sempre a apontar para um ausente.

No texto de Britto (2000), há uma discussão acerca das possíveis práticas interculturais a partir das quais se poderia atribuir a Bishop o papel de intermediária cultural. Dentre elas, destacam-se a correspondência, o livro Brazil editado pela Time-Life, a tradução do livro Minha Vida de Menina, a antologia organizada a partir da poesia modernista e os poemas com temáticas brasileiras. No entanto, Britto (2000) pontua que a autora não falava o português, além de manter uma relação distanciada com a cultura e de nutrir uma visão preconceituosa. Com isso, considera Bishop "[...] a mediadora mais ineficiente e relutante” (BRITTO, 2000, p. 496, tradução nossa). Tal afirmação contrapõe-se às concepções teóricas norteadoras do volume, que toma a linguagem como não transparente ao objeto que intenta representar. Com isso, compreendemos que não há uma verdade sobre o Brasil a ser revelada, assim como pontuou Rocha (2000).

Propomos, desse modo, uma discussão acerca da atuação e produção da autora no Brasil a partir dos conceitos de paradigma indiciário, a fim de observar como seu pensamento contribui para a difusão de discursos a respeito do país. Na função de sujeito dotado de autoridade discursiva, Bishop deve ser considerada um dos agentes que cumpriu, em seu tempo, o papel de divulgadora desta cultura, embora tenha tocado, em alguns momentos, em aspectos espinhosos que podem não gerar sentimento de identificação em muitos brasileiros. Suas representações, aliás, continuam a circular, desenhando, em seus poemas, um Brasil filtrado pela sensibilidade do olhar estrangeiro. 


\section{ESTRANHOS NA HSTÓRIA DA LITERATURA BRASILEIRA: ELIZABETH BISHOP COMO MEDIADORA CULTURAL}

\section{BISHOP E SUA INSERÇÃO NA HISTÓRIA LITERÁRIA BRASILEIRA}

$\mathrm{O}$ arquivo do escritor é materialidade que requer abordagem interdisciplinar, pois a justificativa de sua existência está ancorada em áreas do conhecimento voltadas para a preservação de traços do passado, uma necessidade contemporânea que conduz cada indivíduo, mesmo o anônimo, a preocupar-se com o arquivamento das próprias memórias.

Reúne materiais produzidos em determinada época, com funcionalidade específica para seu criador. No entanto, tais objetos acabam recebendo novas funções para atender ao interesse de uma coletividade (NORA, 1993). Revisitar um arquivo, nessa conjuntura, significa atribuir vida nova à experiência passada à qual imprimimos novas interpretações. O arquivamento pelas mãos do autor, nesse sentido, pode ser desvelado como a morte de uma experiência, mais especificamente, de uma prática escritural. O arquivista, ao reunir e ordenar esse material, faculta acesso a outros pesquisadores, ao mesmo tempo que possibilita novas leituras e interpretações. Por essa razão, tais materiais são reunidos a fim de serem salvaguardados da deterioração imprimida pelo tempo e pelo esquecimento.

$\mathrm{O}$ arquivo em questão viabiliza pensarmos como os manuscritos da autora estrangeira podem dialogar com a história da literatura e cultura brasileira para, desse modo, podermos reafirmar o lugar de Bishop como mediadora cultural, já que, no momento de sua saída do Brasil tanto quanto em momentos recentes, quando seu nome foi indicado para a 18. ${ }^{a}$ Festa Literária Internacional de Paraty, essa posição foi questionada (sobre a qual não pretendemos aqui levantar discussão).

Tais polêmicas fundamentaram-se, principalmente, no posicionamento político de Bishop, conhecido a partir de comentários tecidos em carta. Esses documentos privados, após sua morte, foram publicados no livro One Art e traduzido no Brasil por Britto (BISHOP, 1995). Soma-se a isso a participação em seu ciclo de amizades - na verdade, o de Lota, sua companheira brasileira - composto por ícones da elite carioca, incluindo políticos e artistas.

Diante de tantas controvérsias relacionadas ao nome da autora, pensamos que uma análise de sua obra, incluindo a materialidade inacabada dos manuscritos, permite estabelecer novos diálogos com o papel de mediadora que Bishop ocupou e sua frequente contestação. Para tanto, o paradigma indiciário pode revelar que, embora Bishop tenha apresentado uma visão colonialista e etnocêntrica em relação ao Brasil como afirmou Britto (2000), as imagens 


\section{ESTRANHOS NA HSTÓRIA DA LITERATURA BRASILEIRA: ELIZABETH BISHOP COMO MEDIADORA CULTURAL}

engendradas servem como representativas, no sentido de participarem do imaginário de seus leitores.

Outro conceito que pode ser recuperado é o de circularidade cultural, desenvolvido por Bakhtin e revisitado por Ginzburg (2007) para pensar em um substrato cultural que transita entre os grupos dominantes e os subalternos. No contexto deste artigo, ampliamos a escala desse conceito para pensar em um substrato que circula entre Brasil e Estados Unidos, tendo Bishop como uma mediadora. Trata-se de " [...] relacionamento circular feito de influencias recíprocas, que se movia de baixo para cima, bem como de cima para baixo" (GINZBURG, 2007, p. 13).

Para abordar o papel mediador de Bishop, recorremos ao estudo feito a partir dos rascunhos do livro de viagens Brazil, de autoria de Barbosa (2010), pois realizou um acompanhamento da construção de representações de brasilidade realizadas em um projeto idealizado por Bishop por muito tempo. Ao longo de sua permanência no Brasil, trabalhou nesse projeto artístico e, para isso, realizou viagens por todo o país, a fim de produzir materialidades escrita e fotográfica. Em relação ao livro Brazil, a pesquisa no arquivo da autora tornou-se atividade importante, já que seu formato final sofreu interferência dos editores da Time \& Life, a ponto de resultar em retirada do nome de Bishop como autora a partir da terceira edição.

É relevante notar que seu Brasil ideal envolve lugares remotos, onde se pode encontrar uma vida mais próxima do adjetivado por ela como primitiva. Inclusive, em carta ao amigo Robert Lowell datada de abril de 1960, Bishop menciona que Lota não a acompanhava em suas visitas a esses lugares, aos quais ela mantinha grande interesse.

Semana que vem vou viajar de novo, uma viagem curta - três ou quatro dias - vou a um lugar no litoral onde só se chega de barco - por isso ninguém nunca vai lá, é claro. Dizem que é tudo do século XVIII, perfeitamente conservado - o lugar é bem pequeno - e a maré cobre as ruas todos os dias e depois desce - não tem hotel. A Lota não tem o menor interesse por nada que seja brasileiro ou 'primitivo', você sabe. Ela diz que quando viaja quer mais civilização e não menos - por isso vou com dois vizinhos (BISHOP, 1995, p. 416).

Podemos então afirmar que o estilo de vida da autora dizia mais respeito a uma vida natural, afastada das turbulências de cidades grandes. Suas casas preferidas no Brasil eram a de Petrópolis, local em que podia permanecer isolada em uma fazenda de propriedade de Lota no alto de uma serra ou, ainda, a casa de Ouro Preto, localizada em uma cidade onde a vida simples era preservada. Seu encantamento por essa cidade pode ser atestado em alguns de seus 


\section{ESTRANHOS NA HSTÓRIA DA LITERATURA BRASILEIRA: ELIZABETH BISHOP COMO MEDIADORA CULTURAL}

pronunciamentos: “[...] as pessoas nos lugarejos pobres são absolutamente naturais, e de uma polidez e educação extraordinárias” (BISHOP, 1995, p. 476), e “[...] gosto de Ouro Preto porque lá tudo foi feito ali mesmo, à mão, com pedra, ferro, cobre e madeira. Tiveram que inventar muita coisa - e tudo está em perfeito estado há quase trezentos anos [...]” (BISHOP, 1995, p. 484).

Seu estilo de vida está associado à fuga dos locais onde a intelectualidade ou a vida urbana se estabeleceram. Certamente por isso preferiu viver no Brasil e não em seu país de origem. Quanto a seu estilo de vida no espaço brasileiro, optava por viver em cidades menores. Eram os locais a partir dos quais podia expressar sua arte em maior grandeza.

Segundo Fortuny (2003), embora Bishop tenha evitado um explícito ativismo público na vida e na poesia, ele aparece em camadas menos evidentes de sua obra, a exemplo dos conflitos com a Time-Life em que percebemos uma insatisfação com a visão dos editores em relação ao modo como ela representava a cultura brasileira. No processo de escrita do livro, a Time-Life estava, na realidade, interessada em atender aos gostos de um público de classe média enquanto Bishop se colocava contra tal direcionamento (BARBOSA, 2010).

Nessa mesma perspectiva, Fountain (2003) identifica uma personalidade política que leva a crer que, para a autora, a perspicácia política e a integridade retórica não estão separadas, pois aparecem interligadas de forma que, em seu projeto artístico, tais elementos mantêm uma relação de dependência. É importante não esquecer que dois dos poemas mais belos de Bishop fazem denúncia social e retratam as contradições do Brasil: Pink Dog, ambientado em um momento de carnaval, tem a cadela rosada como personagem de um triste episódio da história brasileira em que mendigos eram jogados no rio da Guarda; e Burglar of Babylon, poema que denuncia a violenta ação policial contra pessoas marginalizadas enquanto as abastadas assistem à cena trágica com binóculos a partir de seus apartamentos.

Parece que a vida no Brasil e o contato com amigos da alta sociedade brasileira (especialmente aqueles envolvidos diretamente com a política), associada com as notícias diárias de violência e barbárie trouxeram, de alguma forma, essa temática para o rol de seus interesses. Em 1966, comentou a respeito de seu posicionamento em relação à política, o qual foi mudando com o passar do tempo: "Sempre me opus ao pensamento político como tal para escritores. Que boa escrita saiu desse período, realmente? [...] Estou mais interessada nos problemas sociais e na política agora do que quando eu tinha 30" (ESTESS; SCHWARTZ, 1999, p. 293, tradução nossa). 


\section{ESTRANHOS NA HSTÓRIA DA LITERATURA BRASILEIRA: ELIZABETH BISHOP COMO MEDIADORA CULTURAL}

O novo território foi capaz de operar mudanças, no sentido de formatar ideias e conceitos. Nesse sentido, podemos afirmar que a viagem e permanência no Brasil funcionou não apenas como deslocamento espaço-temporal, mas propulsionou uma mudança a partir de sua condição de observadora. Aos poucos, foi percebendo com mais acuidade certos aspectos da cultura com a qual entrou em contato, o que leva o sujeito a modificar-se e criar para si identificações não previstas antes da viagem. Desse modo, as percepções registradas por Bishop, em diferentes momentos, atestam que a experiência do deslocamento somente pode revelar aspectos de si e do Outro de forma parcial.

Além disso, os discursos produzidos nunca são inteiramente novos, mas partem de transformações de ideias anteriormente consolidadas. Nos escritos do livro Brazil, podemos destacar a intertextualidade como recurso bastante utilizado em que textos de antigos viajantes e antropólogos deram sustentação às percepções de Bishop a respeito do país. Tal procedimento privilegia uma autoridade discursiva consolidada sobre temáticas brasileiras e, ao mesmo tempo, a reforça.

Encontramos em seus manuscritos referências a autores da literatura de viagem que atuaram como modelos formatadores de sua visão a respeito do povo e cultura do Brasil. Alguns dos nomes são Richard Francis Burton, Maria Graham (Journal of a Voyage to Brazil), Charles Darwin (Voyage de Beagle), além do antropólogo Claude Levi-Strauss (Triste Tropiques) e, ainda, alguns críticos de livros de viagem, como Konrad Guenther (A naturalist in Brazil) e W. B. Greenlee (Cabral's Voyage to Brazil \& Índia).

$\mathrm{Na}$ medida em que outros estrangeiros serviram a Bishop como mediadores culturais, as imagens, já em circulação, foram tanto reforçadas como transformadas a partir da experiência pessoal neste espaço. Assim, a autora reafirmou uma visão de Brasil, apresentando aspectos de novidade sobre essa cultura, o que pode ser investigado a partir de uma metodologia proposta por Ginzburg (2007), a do paradigma indiciário.

Segundo tal paradigma, não é possível conhecer um fenômeno diretamente, mas somente por meio da observação e da análise de indícios, a partir dos quais obtém-se um conhecimento indireto. Para Ginzburg (2007), “[...] se a realidade é opaca, existem zonas privilegiadas - sinais, indícios - que permitem decifrá-la”.

Os indícios que podemos observar nos manuscritos do livro Brazil a partir de um estudo de gênese realizado na dissertação de mestrado de Barbosa (2010) é que há uma bagagem de conhecimento sobre o Brasil apreendida a partir da leitura dos antigos viajantes. No entanto, 


\section{ESTRANHOS NA HSTÓRIA DA LITERATURA BRASILEIRA: ELIZABETH BISHOP COMO MEDIADORA CULTURAL}

muitos dos relatos de Bishop surgem eivados da experiência pessoal em terras brasileiras. As representações tecidas por estrangeiros têm seus contornos modificados por uma experiência pessoal, marcada, a princípio, por um encantamento que, aos poucos, no caso de Bishop, foi se transformando em afetividade. Muitas dessas construções passaram por processamentos inconscientes que mesclam sentimentos contraditórios, o que podemos observar não somente em Brazil, mas no conjunto de sua obra.

Outro ponto a ser considerado a respeito do período em que a autora permaneceu no Brasil diz respeito à formação de identidades, a dela própria e a do espaço representado. Para isso, retomamos o conceito de circularidade cultural relacionado ao fato de que só se define uma identidade a partir da diferença. Nesse sentido, estar em contato com o outro, para Bishop, funcionou como processo de descoberta, no qual pôde experimentar sentimentos diversos em relação ao espaço brasileiro, de apego, de posse e de afetividade, mas, também, de distanciamento e, até mesmo, de aversão. O novo espaço modifica seu visitante, ao mesmo tempo em que é modificado por essa presença estrangeira.

A metodologia do paradigma indiciário associada ao conceito de circularidade possibilitou pensar a respeito do caráter fragmentário da representação. Os significantes que flutuam nos escritos de Bishop, na condição de constructos intelectuais ou estéticos, podem apresentar-se como contraditórios, se levarmos em consideração o conjunto das imagens produzidas no decurso de uma vida. Por exemplo, por vezes, Bishop afirmou ter sido o Brasil o seu único lar e, em outros momentos, apresentou dúvidas em relação a sua posição de expatriada: “[...] mas este aqui não é o meu mundo. Ou é?” (BISHOP, 1995, p. 743).

Nesse contexto, podemos abordar o arquivo como lugar privilegiado para uma abordagem indiciária, já que guardou os vestígios da construção representacional que descortinou uma descoberta de si e do Outro.

$\mathrm{Na}$ contemporaneidade, há uma maior abertura desses arquivos e a consequente circulação de novas representações que reforçam imagens de Brasil já construídas e estereotipadas ou apresentam uma textualidade na qual se imprime a sensibilidade da poeta e, consequentemente, aspectos já conhecidos apresentados de forma inteiramente nova. Em um caso ou em outro, há a circularidade de culturas, processo acionado sempre que se entra em contato com as produções de Bishop a respeito do Brasil.

Isso porque sua vida e identidade foi tecida nos diversos lugares percorridos especialmente no Brasil, onde viveu por aproximadamente vinte anos, e nos Estados Unidos, 


\section{ESTRANHOS NA HSTÓRIA DA LITERATURA BRASILEIRA: ELIZABETH BISHOP COMO MEDIADORA CULTURAL}

onde nasceu e para onde direcionou a voz do eu poético surgido em seus textos. Com o acesso a esses papéis privados e o crescente interesse dos brasileiros e estrangeiros na obra de Bishop, sua identidade de autora que poetizou o povo, os espaços e a cultura brasileira continua a ser construída a cada leitura de seus textos.

$\mathrm{O}$ estudo do arquivo sob o viés do paradigma indiciário revelou que a produção de Bishop sobre temáticas brasileiras pode ser descortinada de três formas: como uma maneira de moldar e entender a própria identidade, a qual só poderia ser problematizada a partir de traços distintivos em relação ao outro; como um reforço das representações realizadas por outros viajantes; e, principalmente, como discurso que integra um mosaico de representações acerca de um Brasil que, como apontou Drummond (2013) em seu poema Hino Nacional, não existe.

Assumir os papéis de viajante e de estrangeira se tornou, para Bishop, o caminho para a busca de si mesma. Através da imagem do Outro, ela foi capaz de descobrir aspectos do eu ainda não conhecidos. Alguns vestígios ficaram registrados em seus escritos, conforme ia formatando uma identidade para si. Travisano (1989) afirma que o importante para Bishop sempre foi o objeto diante de seus olhos. Os indícios deste estudo supõem que Bishop foi em busca de elementos culturais que considerava serem genuinamente brasileiros.

Diante do exposto, consideramos que a autora cumpriu seu papel de mediadora cultural e, portanto, seu nome deve figurar entre os estrangeiros que leram este país. As representações engendradas por ela e por outros autores, sejam brasileiros ou estrangeiros, não coincidem com o Brasil, assim como afirmou Rocha (2000). No entanto, servem como influências para que cada leitor possa compor uma identidade nacional não fixa, que se altera ao longo do tempo e em contato com novos textos.

\section{REFERÊNCIAS}

BARBOSA, Elisabete da Silva. Identidades entrelaçadas: uma visita ao Brazil, de Elizabeth Bishop. 2010. 172 f. Dissertação (Mestrado em Linguagens) - Programa de PósGraduação em Linguagem da Universidade do Estado da Bahia, Salvador, 2010.

BHABHA, Homi. O local da cultura. Belo Horizonte: Editora UFMG, 2007.

BISHOP, Elizabeth \& The Editors of LIFE. Brazil. New York: Time Incorporated, 1967.

BISHOP, Elizabeth. Uma arte: as cartas de Elizabeth Bishop. Trad. e org. Paulo Henriques Britto. São Paulo: Companhia das letras, 1995. 
BLOOM, Harold. O cânone ocidental. Trad: Marcos Santarrita. Rio de Janeiro: Ponto de Leitura, 2010.

BRITTO, Paulo Henriques. Elizabeth Bishop as Cultural Intermediary. In: ROCHA, João Cezar de Castro (ed.). Brazil 2001: A revisionary history of Brazilian Literature and Culture. Portuguese Literary Cultural Studies 4/5. Massachusetts, spring / fall 2000, p. 489506.

CANDIDO, Antonio. Formação da literatura brasileira: momentos decisivos. Rio de Janeiro: Ouro sobre azul, 2012 [1959].

CHAUÍ, Marilena. Brasil. Mito fundador e sociedade autoritária. São Paulo: Editora Fundação Perseu Abramo, 2000.

COUTINHO, Afrânio (org.). A literatura no Brasil - introdução geral. Vol. 1. São Paulo: Global, 2004 [1968].

DENIS, Ferdinand. Resumo da história literária de Portugal seguido do resumo da história literária do Brasil. Trad. Regina Zilberman. Rio de Janeiro: Edições Macunaíma, 2018 [1826].

DRUMMOND DE ANDRADE, Carlos. Hino Nacional. In: Brejo das Almas. São Paulo: Companhia das Letras, 2013.

ESTESS, Sybil P; SCHWARTZ, Lloyd. Elizabeth Bishop and her art. Michigan: University of Michigan Press, 1999.

FORTUNY, Kim. Elizabeth Bishop. The art of travel. Colorado: University Press of Colorado, 2003.

FOUNTAIN, Gary. Elizabeth Bishop's "idea of the place”. Collecting, Culture, and the Aesthetics of National Identity. In: ALMEIDA, Sandra Regina; GONÇALVES, Glaucia. REIS, Eliana (orgs.). The art of Elizabeth Bishop. Belo Horizonte: UFMG, 2002, p. $259-271$

GINZBURG, Carlo. Mitos, emblemas, sinais. Morfologia e história. Trad: Frederico Carotti. São Paulo: Companhia das Letras, 2007.

NORA, Pierre. Entre memória e história: a problemática dos lugares. Trad. Yara Khoury.

Revista do Programa de Estudos em História e do Departamento de História da PUCSP, São Paulo, n. 10, p. 07-28, dez. 1993. Disponível em:

<https://revistas.pucsp.br/revph/article/view/12101>. Acesso: 22 mar. 2021.

RICOEUR, Paul. A memória, a história, o esquecimento. Campinas: Ed. Unicamp, 2007.

ROCHA, João Cezar de Castro (ed.). Brazil 2001: A revisionary history of Brazilian Literature and Culture. Portuguese Literary Cultural Studies 4/5. Massachusetts, spring / fall 2000. 
ESTRANHOS NA HSTÓRIA DA LITERATURA BRASILEIRA: ELIZABETH BISHOP COMO MEDIADORA CULTURAL

ROMERO, Sílvio. História da literatura brasileira. Rio de Janeiro: Imago, 2001.

TRAVISANO, Thomas. Elizabeth Bishop: Her artistic development. Charlottesville: University Press of Virginia, 1989.

VERÍSSIMO, José. História da literatura brasileira: de Bento Teixeira (1601) a Machado de Assis (1908). $1^{\circ}$. milheiro. Rio de Janeiro: Livraria Francisco Alves \& Cia, 1916. 